PREPARED FOR APJ

Preprint typeset using $\mathrm{LT}_{\mathrm{E}} \mathrm{X}$ style emulateapj v. 5/2/11

\title{
EXPECTATION ON OBSERVATION OF SUPERNOVA REMNANTS WITH THE LHAASO PROJECT
}

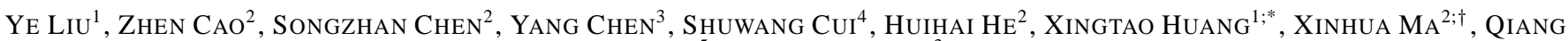 \\ YUAN $^{5}$ AND XIAO ZHANG ${ }^{3}$ \\ ON BEHALF OF THE LHAASO COLLABORATION
}

Prepared for ApJ

\begin{abstract}
Supernova remnants (SNRs) are believed to be the most important acceleration sites for cosmic rays (CRs) below $\sim 10^{15} \mathrm{eV}$ in the Galaxy. High energy photons, either directly from the shocks of the SNRs or indirectly from the interaction between SNRs and the nearby clouds, are crucial probes for the CR acceleration. Big progresses on observations of SNRs have been achieved by space- and ground-based $\gamma$-ray facilities. However, whether $\gamma$-rays come from accelerated hadrons or not, as well as their connection with the CRs observed at Earth, remains in debate. Large High Altitude Air Shower Observatory (LHAASO), the next generation experiment, is designed to survey the northern part of the very high energy $\gamma$-ray sky from $\sim 0.3 \mathrm{TeV}$ to $\mathrm{PeV}$ with the sensitivity of $\lesssim 1 \%$ of the Crab nebula flux. In this paper, we indicate that LHAASO will be dedicated to enlarging the $\gamma$-ray SNR samples and improving the spectral and morphological measurements. These measurements, especially at energies above $30 \mathrm{TeV}$, will be important for us to finally understand the CR acceleration in SNRs.
\end{abstract}

Subject headings: acceleration of particles - cosmic rays - gamma rays

\section{INTRODUCTION}

Cosmic rays (CRs), discovered more than one century ago, represent the most energetic, and, to some degree, fundamental aspect of the Universe. Nuclei constitute dominantly $(\sim 99 \%)$ CRs, and electrons contribute the rest $\sim 1 \%$. The energy spectrum of CRs observed at Earth is well described by a single power-law up to energies of a few $\mathrm{PeV}$, above which the spectrum steepens to form the so-called "knee" (Horandel 2003; Blumer et al. 2009). It is general believed that CRs below the "knee" originate from the Galaxy, due to a simple argument that the Galactic magnetic field would effectively confine such particles. However, their acceleration sites and the acceleration mechanism remain open questions.

The deflection of charged CRs in the Galactic magnetic field makes it very difficult to identify the sources of CRs. High energy $\gamma$-rays, produced by either the collision between $\mathrm{CR}$ hadrons and the ambient medium or the interaction between CR leptons and the interstellar radiation field and/or the medium, are thus more powerful to probe the sources of CRs. With quick development of the detection technology, great progresses have been achieved in high energy $\gamma$ ray astronomy during the past two decades. The satelliteborne instrument Fermi Large Area Telescope (Fermi-LAT), launched in 2008, discovered over 3000 sources in GeV energies (Acero et al. 2015a). In TeV energies, more than 160 $\gamma$-ray sources ${ }^{1}$ have been firmly detected by the ground-based

\footnotetext{
* Corresponding author: huangxt@sdu.edu.cn

$\dagger$ Corresponding author: maxh@ihep.ac.cn

${ }^{1}$ School of Physics \& Key Laboratory of Particle Physics and Particle Irradiation(MOE), Shandong University, Jinan 250100, China

${ }^{2}$ Institute of High Energy Physics, Chinese Academy of Sciences, Beijing 100049, China

${ }^{3}$ Department of Astronomy, Nanjing University, Nanjing 210093 , China

${ }^{4}$ The College of Physics Science and Information Engineering, Hebei Normal University, Shijiazhuang, 050016, China

${ }^{5}$ Department of Astronomy, University of Massachusetts, 710 North Pleasant St., Amherst, MA, 01003, U.S.A
}

detectors, including Imaging Atmospheric Cherenkov Telescopes (IACTs) such as H.E.S.S., MAGIC, and VERITAS, and extensive air shower (EAS) arrays such as Tibet-AS $\gamma$, Milagro, and ARGO-YBJ. Most of these $\gamma$-ray sources are likely to be leptonic, including pulsars, pulsar wind nebulae (PWN), binary systems and so on (e.g., Aharonian et al. 2006; Chen 2013; Bartoli et al. 2015). Clear evidence of the sources of hadronic CRs is observationally rare.

It is widely believed that supernova remnants (SNRs) could efficiently accelerate particles at the shock front where the expanding supernova ejecta encounter the surrounding medium (Bell 1978a,b; Drury et al. 1994). The wellestablished diffusive shock acceleration (DSA) theory (Drury 1983; Blandford et al. 1987) predicts a power-law spectrum of the accelerated particles with index of $\sim 2$ (Holder 2012; Schure et al. 2012), well consistent with the radio observations of $\mathrm{SNRs}^{2}$, as well as the locally observed CR spectrum accounting for the propagation effect. Moreover, the importance of magnetic field amplification by the accelerated particles themselves has been increasingly recognized, which is expected to play a particularly important role in explaining the CRs around the knee region ( $\sim \mathrm{PeV})($ Bell 2004).

"\|l Based on the multi-wavelength observations of SNRs, a class of SNRs have been suggested to be efficient electron accelerators, e.g., RX J1713.7-3946 (Ellison et al. 2010; Abdo et al.2011; Li et al.2011; Yuan et al. 2011), RX J08524622 (Tanaka et al. 2011), RCW 86 (Yuan et al. 2014), HESS J1731-347 (Yang et al. 2014; Acero et al. 2015b), and SN 1006 (Acero et al. 2010; Araya et al. 2012; Acero et al. $2015 b)$. On the other hand, Fermi-LAT collaboration reported the detection of the characteristic pion-decay bump in the GeV $\gamma$-ray spectra of two ancient SNRs, IC 443 and W44, which are interacting with molecular clouds (Ackermann et al. 2013). An earlier detection of hard sub$\mathrm{GeV}$ spectrum of W44 by AGILE was also explained to be

1 http://tevcat.uchicago.edu/

2 http://www.mrao.cam.ac.uk/surveys/snrs/ 
hadronic origin (Giuliani et al. 2011). These observations might give direct evidence for hadronic CR acceleration in SNRs (Ackermann et al. 2013). However, the role of electron bremsstrahlung in this energy range is still unclear, due to the poorly constrained sub-GeV electron spectrum.

The observations of very high energy (e.g., $>100 \mathrm{TeV}) \gamma-$ ray emission from SNRs will provide a complimentary test of the leptonic/hadronic origin of the $\gamma$-rays. It is expected that electrons will be difficult to be accelerated sufficiently to high energies due to the strong synchrotron and/or inverse Compton cooling. A rough estimate of the synchrotron cooling gives the cooling energy, $E_{c} \sim 10(B / \mu \mathrm{G})^{-2}\left(T / 10^{3} y r\right)^{-1}$ $\mathrm{PeV}$, above which electrons will cool down. For a typical magnetic field strength of tens of $\mu \mathrm{G}$ and an age of $10^{3} \mathrm{yr}$, $E_{c} \lesssim 100 \mathrm{TeV}$. The acceleration of high energy electrons will be even more difficult for old SNRs. Furthermore, the inverse Compton radiation efficiency of very high energy electrons will be suppressed due to the Klein-Nishina effect, making the contribution to very high energy $\gamma$-rays more difficult. It is thus expected that $\gamma$-ray emission above $100 \mathrm{TeV}$ from SNRs (especially the old ones), either from shock-crushed dense clouds (e.g. Blandford \& Cowie 1982; Uchiyama et al. 2010) or from the adjacent MCs illuminated by escaping protons (Li \& Chen 2010, 2012), will strongly support the nuclei acceleration. Besides, the $\gamma$-ray observation above 100 $\mathrm{TeV}$ is also expected to give a constraint on the cutoff energy of shock-accelerated proton, which can help to make a distinction between the theoretical models (Zhang \& Chen 2016). These become the major motivations that we propose the next generation, $\mathrm{km}^{2}$ scale $\mathrm{CR} / \gamma$-ray observatory: the Large High Altitude Air Shower Observatory (LHAASO; $\mathrm{CaO} 2010 ; \mathrm{He} 2015)$. With the large effective area $\left(\sim \mathrm{km}^{2}\right)$ and large field-of-view ( $\sim 2 \mathrm{sr}$ ), as well as the high CR rejection power $(\sim 1 \%)$, LHAASO is dedicated to surveying the northern very high energy $\gamma$-ray sky with sensitivity of $\sim 1 \%$ of the Crab nebula flux. LHAASO is expected to not only discover many new sources, but also improve the spectral and morphological measurements of known sources to the highest achievable energies (Cui et al. 2014; Zhao et al. 2016).

In this work we study the perspective of observing very high energy $\gamma$-ray emission from SNRs with LHAASO. We intend to understand the physical potential of LHAASO on the longstanding problem of the origin of hadronic CRs. The rest of this paper is organized as follows. We briefly introduce the design of LHAASO detectors and their performances on $\gamma$ ray detections in Sec. 2. The expected performance of SNR observations with LHAASO based on the current $\mathrm{GeV}$ and/or $\mathrm{TeV} \gamma$-ray observations is presented in Sec. 3. We will pay special attention on the spectral measurements to $>30 \mathrm{TeV}$ energies. We conclude our work with some discussions in Sec. 4.

\section{THE LHAASO PROJECT AND ITS PERFORMANCE}

LHAASO $\left(100.01^{\circ} \mathrm{E}, 29.35^{\circ} \mathrm{N}\right)$ is a recently approved, new generation EAS experiment which will be built at $4410 \mathrm{~m}$ above the sea level near the Daocheng village, in Sichuan province, China. LHAASO is a hybrid extensive air shower (EAS) array covering an area of $1 \mathrm{~km}^{2}$ (KM2A), water Cherenkov detector array (WCDA), and wide field-of-view imaging Cherenkov telescope array (WFCTA). The major scientific goals of LHAASO are: 1) precisely measuring the spectra of $\gamma$-ray sources at high energy range for studying the acceleration and propagation of CRs, 2) deeply survey-

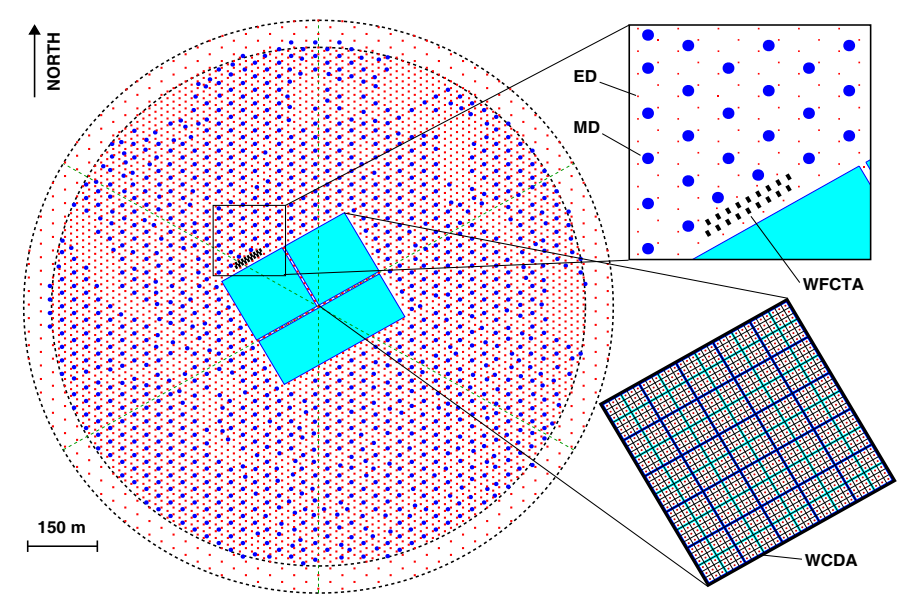

Figure 1. Schematic plot of LHAASO detectors.

ing the very high energy $\gamma$-ray sky (Dec. from $-10^{\circ}$ to $70^{\circ}$ ) for exploring the high energy radiation mechanism, and 3) effectively searching for dark matter and the new physics (Cao 2010; He 2015). The setup of LHAASO (see Fig. 1) and its performance are as follows.

- KM2A, with an effective area of $1 \mathrm{~km}^{2}$, is composed of 5195 scintillator electron detectors (EDs) with $1 \mathrm{~m}^{2}$ each and a spacing of $15 \mathrm{~m}$, and 1171 muon detectors (MDs) with 36 $\mathrm{m}^{2}$ each and a spacing of $30 \mathrm{~m}$. The detector performance are discussed in (Cui et al. (2014); He (2015)). At $10 \mathrm{TeV}$, the effective area of KM2A can reach about $0.3 \mathrm{~km}^{2}$, the angular resolution is about $0.86^{\circ}$, and the energy resolution for $\gamma$-rays is about $42 \%$. The corresponding values are $0.8 \mathrm{~km}^{2}, 0.5^{\circ}$, $33 \%$ at $30 \mathrm{TeV}$, and $0.9 \mathrm{~km}^{2}, 0.3^{\circ}, 20 \%$ at $100 \mathrm{TeV}$ respectively. With the large area of MD array, KM2A will reject the hadronic shower background at a level of $10^{-4}$ at $50 \mathrm{TeV}$ and even $10^{-5}$ at higher energies, so that $\gamma$-rays samples can reach background free above $100 \mathrm{TeV}$. The highest sensitivity of $\mathrm{KM} 2 \mathrm{~A}$ is $\sim 1 \%$ of the Crab nebula flux in the energy range of $50-100 \mathrm{TeV}$ for one year observation.

- WCDA, with an effective area of $78,000 \mathrm{~m}^{2}$ and 3000 units, will be built at the center of KM2A. The size of one unit is about $5 \mathrm{~m} \times 5 \mathrm{~m}$, and the effective water depth is about 4.4 $\mathrm{m}$. Each unit is separated by plastic curtains vertically hung in water, and contains a photomultiplier tube anchored at the center of the cell bottom. The detector performance are discussed in (Yao et al. (2009, 2011)). At $0.5 \mathrm{TeV}$, the effective area can reach $3,000 \mathrm{~m}^{2}$, the angular resolution is $0.6^{\circ}$ and the energy resolution for $\gamma$-rays is $95 \%$. The corresponding values are $10,000 \mathrm{~m}^{2}, 0.4^{\circ}, 90 \%$ at $1 \mathrm{TeV}$, and $50,000 \mathrm{~m}^{2}, 0.2^{\circ}$, $60 \%$ at $10 \mathrm{TeV}$. The highest sensitivity is $\sim 1 \%$ of the Crab nebula flux at the energy around $3 \mathrm{TeV}$.

- WFCTA, made up of 12 telescopes, will be built near WCDA. These detectors are to measure the energy spectral of different composition of CRs up to the second knee, less relevant to the $\gamma$-ray detection as discussed in this work.

\section{EXPECTATION ON OBSERVATION OF SNRS WITH LHAASO}

\subsection{Simulation of observing a $\gamma$-ray source}

To estimate LHAASO observation for a $\gamma$-ray source, both $\gamma$-ray and cosmic ray background samples are produced by Corsika 6.600. The detector response of WCDA is simulated based on GEANT4 program developed by the Milagro collaboration (Yao et al. 2009). For KM2A, a fast simulation pre- 


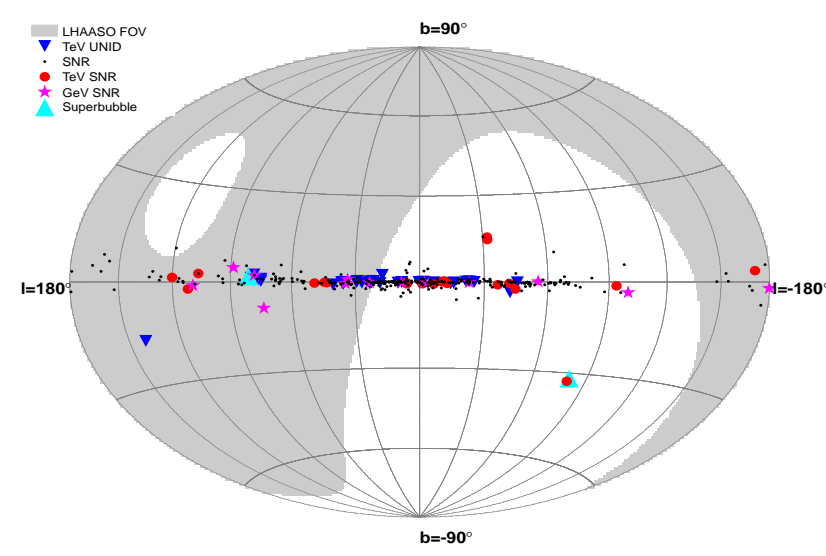

Figure 2. Locations of SNRs and unidentified $\mathrm{TeV} \gamma$-ray sources in Galactic coordinates, compared with the field-of-view of LHAASO (grey region). Black dots represent SNRs from Green ${ }^{1}$, red filled circles and magenta stars show $\mathrm{TeV}$ and $\mathrm{GeV} \gamma$-ray SNRs ${ }^{1}$ (Acero et al. 2015a), blue triangles represent the unidentified $\mathrm{TeV} \gamma$-ray sources, and cyan triangles represent two superbubbles which were detected in $\mathrm{TeV} \gamma$-ray bands. (A color version of this figure is available in the online journal.)

sented in (Cui et al. 2014) is adopted. The simulation events are sampled in energy range from $100 \mathrm{GeV}$ to $10 \mathrm{PeV}$ with the zenith angle less than $45^{\circ}$.

In order to obtain the signals $\left(\mathrm{N}_{\gamma}\right)$ and background $\left(\mathrm{N}_{b g}\right)$ within a specific space angle center on the source, we have traced the source by means of a complete transit, i.e., 24 hours of observation. We only adopt the proton to simulate the cosmic ray background, while we adjusted the flux to the all particles flux (Ivanenko et al. 1988). We use Eq.(17) of $\mathrm{Li}$ and $\mathrm{Ma}(\mathrm{Li} \& \mathrm{Ma}(1983)$ ) to calculate the significance.

\subsection{Detectability of SNRs and SNR candidates}

According to Dave Green's Galactic SNR catalogue ${ }^{1}$ |, 294 SNRs have been detected up to now. Most of these SNRs are detected in low energy bands. In $\mathrm{GeV}$ energies, the FermiLAT collaboration reported their first SNR catalog based on three year's survey data, in which 12 firm identifications and 11 possible associations with SNRs were found (Acero et al. 2015a). In TeV energies, there have been 23 SNRs or SNR candidates detected up to now, 10 of which are also $\mathrm{GeV} \gamma$ ray emitters ${ }^{2}$. . Furthermore, there are 34 unidentified $\mathrm{TeV}$ $\gamma$-ray sources which do not have clear counterparts in other wavelengths. Different from the Fermi unidentified sources which are expected to be dominately constituted by active galactic nuclei (Mao \& Yu 2013), most of the unidentified $\mathrm{TeV}$ sources are located in the Galactic plane (see Fig. 2) and could be potential SNRs. Fig. 2 illustrates the locations of those sources (symbol) and their visibility by LHAASO (shaded region). In total, 92 out of 294 SNRs in Green Catalog, $6 \mathrm{GeV}$ SNRs or SNR candidates, $2 \mathrm{TeV}$ SNRs and 6 $\mathrm{GeV}-\mathrm{TeV}$ SNRs are in the field of view of LHAASO. Besides, $17 \mathrm{TeV}$ unidentified sources locate in the field of view of LHAASO.

Based on the current $\mathrm{GeV} / \mathrm{TeV}$ observations of SNRs and SNR candidates, we estimate their detectability by LHAASO. The $\gamma$-ray spectrum of each source is assumed to be an expo-

\footnotetext{
1 http://www.mrao.cam.ac.uk/surveys/snrs/

2 http://tevcat.uchicago.edu/
}

nentially cutoff power-law form

$$
\frac{d N}{d E}=J_{0}(E / \mathrm{TeV})^{-\alpha} \exp \left(-E / E_{\text {cut }}\right)
$$

where $J_{0}$ is the flux at $1 \mathrm{TeV}, \alpha$ is the source spectral index, and $E_{\text {cut }}$ is the cutoff energy. We fit the observational data to find the spectral parameters for each source. For the $\mathrm{GeV}$ only sources and some $\mathrm{TeV}$ sources, whose cutoff energies can not be well determined, we assume $E_{\mathrm{cut}}=30$ and $100 \mathrm{TeV}$, respectively.

We simulate the observation of each source and calculate its expected significance for 5 years sky survey of LHAASO. For $\mathrm{GeV}-\mathrm{TeV}$ and TeV SNRs, the expected significance above 10 $\mathrm{TeV}$ under cutoff energy $30 \mathrm{TeV}$ and $100 \mathrm{TeV}$ are presented in Table 1. 6 SNRs can been detected by LHAASO with significance greater than $5 \sigma$ if the cutoff energy is $100 \mathrm{TeV}$. The significance will decrease if the cutoff energy is lower, while, there still are 5 SNRs can been detected by LHAASO if the cutoff energy is $30 \mathrm{TeV}$. This is the key to confirm whether the SNR is hadronic origin or not, shows that the observation level of LHAASO is sensitive to give an judgement on the acceleration mechanism above $30 \mathrm{TeV}$.

Morever, it has been found that some SNRs could emitted $\mathrm{TeV} \gamma$-rays while in $\mathrm{GeV}$ energy band there was no observation results, like G106.3+2.7 and HESS J1912+101. G106.3+2.7 was first observed by DRAO at radio energy range (Joncas et al. 1990). In 2000, Pineault \& Joncas confirmed the object as a SNR, with an estimated age of 1.3 Myr and distance of $12 \mathrm{kpc}$ (Pineault et al. 2000). The pulsar PSR J2229+6114 is located at the northern edge of the remnant's head and it is associated with boomerang-shaped radio and X-ray emitting wind nebula. At GeV energy band, the EGRET source 3EG J2227+6122 is compatible with the pulsar position, as well as the main bulk of the radio remnant (Hartman et al. 1999). And at TeV energy band, VERITAS reported the total flux from the SNR G106.3+2.7 above 1 $\mathrm{TeV}$ is about $\sim 5 \%$ of the Crab Nebular in 2009 Acciari et al. 2009b). HESS J1912+101 is plausibly associated with the PSR J1913+1011, which is detected by H.E.S.S. experiment. The integral flux between $1-10 \mathrm{TeV}$ is $10 \%$ of the Crab Nebula and the measured energy spectrum can be described by a power-law with a photon index $\sim 2.7$. From the current observation on these two TeV SNRs, we can conclude that LHAASO might discover a number of SNRs compared to conservative predictions based on the current SNR catalogs.

\subsection{Case studies}

In this subsection we choose several GeV-TeV SNRs as examples to explore in more details the spectral measurements of their very high energy $\gamma$-ray emissions by LHAASO, and discuss the physical implications of these new measurements. Different from that in $\S 3.2$ whose purpose is a rough estimate of a big sample, we adopt a physically motivated model, either leptonic or hadronic, to characterize the $\gamma$-ray emission of these sources. In order to better fit the observed $\gamma$-ray data, we assume an exponentially cutoff broken power-law form of the injection spectrum of accelerated particles

$$
F(E) \propto \exp \left(-E / E_{c}\right) \times\left\{\begin{array}{l}
E^{-\alpha_{1}}, E<E_{\mathrm{br}} \\
E^{-\alpha_{2}}, E \geq E_{\mathrm{br}}
\end{array},\right.
$$

where $E_{\mathrm{br}}$ and $E_{c}$ are the break energy and cutoff energy, $\alpha_{1}$ and $\alpha_{2}$ are the spectral indices below and above $E_{\mathrm{br}}$ respectively. Based on the current experiment data, both 
Table 1

Table of a selection of known GeV-TeV and TeV SNRs shown with the expected significance above $10 \mathrm{TeV}$ using five years Monte Carlo simulation datas of LHAASO, assuming the sources are under the specific hypotheses for the energy spectrum. ("-" means the significance is less than $5 \sigma$.)

\begin{tabular}{|c|c|c|c|c|c|c|c|c|}
\hline Name & Classification & "R.A. & Dec. & $\overline{\alpha \alpha}$ & $\begin{array}{c}J_{0} \\
\left(\mathrm{TeV}^{-1} \mathrm{~cm}^{-2} \mathrm{~s}^{-1}\right)\end{array}$ & $\begin{array}{c}\sigma \\
\left(E_{\text {cut }}=100 \mathrm{TeV}\right)\end{array}$ & $\begin{array}{c}\sigma^{\prime} \\
\left(E_{\text {cut }}=30 \mathrm{TeV}\right)\end{array}$ & Ref. \\
\hline Tycho(a) & GeV-TeV & $00^{h} 25^{m} 18^{s}$ & $+64^{\circ} 09^{\prime}$ & $2.92 \pm 0.46$ & $2.2 \times 10^{-13}$ & - & - & 1 \\
\hline Tycho(b) & GeV-TeV & $00^{h} 25^{m} 18^{s}$ & $+64^{\circ} 09^{\prime}$ & $1.95 \pm 0.51$ & $1.70 \times 10^{-13}$ & 11.62 & 5.20 & 2 \\
\hline IC 443 & $\mathrm{GeV}-\mathrm{TeV}$ & $06^{h} 17^{m} 00^{s}$ & $+22^{\circ} 30^{\prime}$ & $2.99 \pm 0.38$ & $8.38 \times 10^{-13}$ & 5.46 & - & 3 \\
\hline W49B & GeV-TeV & $19^{h} 11^{m} 08^{s}$ & $+09^{\circ} 06^{\prime}$ & $3.1 \pm 0.3$ & $2.3 \times 10^{-13}$ & - & - & 4 \\
\hline HESS J1912-101 & $\mathrm{TeV}$ & $19^{h} 12^{m} 49^{s}$ & $+10^{\circ} 09^{\prime}$ & $2.7 \pm 0.2$ & $3.5 \times 10^{-12}$ & 59.63 & 28.02 & 5 \\
\hline W51C & GeV-TeV & $19^{h} 23^{m} 50^{s}$ & $+14^{\circ} 06^{\prime}$ & $2.58 \pm 0.07$ & $9.7 \times 10^{-13}$ & 31.44 & 14.87 & 6 \\
\hline G106.3+2.7 & $\mathrm{TeV}$ & $22^{h} 27^{m} 59^{s}$ & $+60^{\circ} 52^{\prime}$ & $2.29 \pm 0.33$ & $1.42 \times 10^{-12}$ & 57.43 & 21.10 & 7 \\
\hline Cassiopeia A & $\mathrm{GeV}-\mathrm{TeV}$ & $23^{h} 23^{m} 26^{s}$ & $+58^{\circ} 48^{\prime}$ & $2.3 \pm 0.2$ & $7.3 \times 10^{-13}$ & 26.51 & 10.20 & 8 \\
\hline
\end{tabular}

Notes: Columns from left to right are: source name, classification, R.A., Declination, spectral index, flux normalization, cutoff energy, expected significance by LHAASO (for $E_{\text {cut }}=100 \mathrm{TeV}$, if the cutoff energy has not been measured), expected significance by LHAASO for $E_{\text {cut }}=30 \mathrm{TeV}$, and the references of the measurements.

References: (1) Park et al. (2015); (2) Acciari et al. (2011); (3) Acciari et al. (2009a); (4) Francois et al. (2011); (5) Aharonian et al. (2008b); (6) Aleksic et al. (2012); (7) Acciari et al. (2009b); (8) Albert et al. (2007b);

Table 2

The leptonic models parameters for SNRs.

\begin{tabular}{|c|c|c|c|c|c|c|c|}
\hline \hline & $\begin{array}{c}\mathrm{d} \\
(\mathrm{kpc})\end{array}$ & $\begin{array}{c}\mathrm{n} \\
\left(\mathrm{cm}^{-3}\right)\end{array}$ & $\alpha 1$ & $\alpha 2$ & $\begin{array}{c}\mathrm{E}_{b r} \\
(\mathrm{GeV})\end{array}$ & $\begin{array}{c}\mathrm{W}_{e} \\
(\mathrm{erg})\end{array}$ & Ref \\
\hline Tycho & 3.0 & 10 & 2.03 & 2.69 & 0.418 & $2.92 \times 10^{48}$ & 9,10 \\
\hline Cassiopeia A & 3.4 & 10 & 1.03 & 2.81 & 6.38 & $6.18 \times 10^{48}$ & 11 \\
\hline IC 443 & 2.0 & 100 & 1.92 & 3.00 & 12.37 & $3.63 \times 10^{48}$ & 12 \\
\hline W51C & 6.0 & 100 & 1.68 & 2.77 & 4.49 & $2.07 \times 10^{49}$ & 13 \\
\hline \hline
\end{tabular}

Notes: $\mathrm{W}_{e}$ is the total energy of electrons.

References: (9) Giordano et al. (2012); (10) Zhang et al. (2013); (11) Abdo et al. (2010b); (12) Yuan et al. (2012); (13) Abdo et al. (2009b);

Table 3

The hadronic models parameters for SNRs.

\begin{tabular}{|c|c|c|c|c|c|c|}
\hline \hline & $\begin{array}{c}\mathrm{d} \\
(\mathrm{kpc})\end{array}$ & $\begin{array}{c}\mathrm{n} \\
\left(\mathrm{cm}^{-3}\right)\end{array}$ & $\alpha 1$ & $\alpha 2$ & $\begin{array}{c}\mathrm{E}_{b r} \\
(\mathrm{GeV})\end{array}$ & $\begin{array}{c}\mathrm{W}_{p} \\
(\mathrm{erg})\end{array}$ \\
\hline Tycho & 3.0 & 10 & 1.21 & 2.36 & 1.12 & $1.07 \times 10^{49}$ \\
\hline Cassiopeia A & 3.4 & 10 & 1.22 & 2.41 & 21.72 & $5.82 \times 10^{49}$ \\
\hline \hline
\end{tabular}

Notes: $\mathrm{W}_{p}$ is the total energy of protons.

leptonic and hadronic scenarios may still remain valid if the $\gamma$-ray spectrum has a cutoff between $10 \mathrm{TeV}$ and 100 $\mathrm{TeV}$ (Slane et al. 2014; Zirakashvili et al. 2014; Abdo et al. $2010 \mathrm{~b})$. Because of the shorter cooling time of electrons, the $\gamma$-ray emitting electrons can be hardly accelerated up to 100 $\mathrm{TeV}$. While there is no strong limit on hadronic acceleration based on the theory or current experiment. Thus, in this paper, we assume $E_{c}^{e} \approx 50 \mathrm{TeV}$ (Yuan et al.2012) in the leptonic model, and $E_{c}^{p}=\infty$ in the hadronic model which means that they can be accelerated to energy above the knee.

\subsubsection{Young SNRs: Tycho and Cassioperia A}

The relatively young SNRs are believed to be the major sites to accelerate CRs up to $10^{15} \mathrm{eV}$, and the detection and identification of hadronic $\gamma$-rays directly from young SNRs would be a straightforward test of acceleration of protons and nuclei.

Tycho, which appeared in 1572 (Hanbury et al. 1953), has been observed from radio to $\mathrm{TeV} \gamma$-ray band (Dickel et al.
1991; Hwang et al. 2002; Bamba et al. 2005; Stroman et al. 2009; Katsuda et al. 2010; Acciari et al. 2011). At the GeV range, Fermi-LAT reported a $5 \sigma$ detection of $\mathrm{GeV} \gamma$-rays emission from Tycho, which can be described by a powerlaw with a photon index $2.3 \pm 0.2$ (Giordano et al. 2012). At the TeV range, VERITAS observed that the total flux of Tycho above $1 \mathrm{TeV}$ is $\sim 0.9 \%$ of Crab Nebula and the spectrum index between $1 \mathrm{TeV}$ and $10 \mathrm{TeV}$ is about $1.95 \pm 0.51$ in 2011 . But in 2015, the spectrum index is $2.92 \pm 0.46$ (Acciari et al. 2011; Park et al. 2015). If the spectral index is about 2 up to $10 \mathrm{TeV}$ as the VERITAS reported in 2011, it implies that the corresponding spectrum of primary protons continues without a significant steepening or a cutoff to at least several hundred TeV (Kelner et al. 2006; Aharonian et al. 2013).Due to the large uncertainties of the data sets of Fermi and VERITAS, the energy spectrum from $\mathrm{GeV}$ to $\mathrm{TeV}$ can be described by a broad range of function which is not enough to explain the high energy $\gamma$-ray emission. 
Cassioperia A (Cas A), appeared in the sky in 1680, is the youngest of the historical Galactic SNRs (Abdo et al. 2010b; Acciari et al. 2010). It is one of the best studied objects with both thermal and non-thermal broad-band emission ranging from radio wavelengths to $\mathrm{TeV} \gamma$-rays (Aharonian et al. 2001; Albert et al. 2007b; Acciari et al. 2010; Yuan et al. 2013). $\mathrm{TeV} \gamma$-ray observations revealed a rather modest $\gamma$-ray flux, compared to the synchrotron radio through $\mathrm{X}$-ray emission, which further strengthens the argument for a rather high magnetic field. In the GeV range, Fermi-LAT observation suggests that leptonic model can not fit the turnover well at low energy because the bremsstrahlung component that is dominant over IC below $1 \mathrm{GeV}$ has a steep spectrum, and hadronic emission describing the $\gamma$-ray spectrum by a broken powerlaw is preferred. However, because the observed $\mathrm{TeV} \gamma$-ray fluxes have large statistical uncertainties, it can not be judged yet whether the $\mathrm{TeV} \gamma$-rays are generated by interactions of accelerated protons and nuclei with the ambient gas or by electrons through bremsstrahlung and inverse Compton scattering. And the maximum energy of the observed $\mathrm{TeV} \gamma$-ray is only several $\mathrm{TeV}$, the question whether Cas A accelerates particles to $\mathrm{PeV}$ energy is still open.

At the LHAASO site, the effective observation time is 6.2 hours per day for Tycho and 6.8 hours per day for Cas A with zenith angle less than $45^{\circ}$. Tycho culminates with a zenith angle of $34^{\circ}$ and Cas A culminates with a zenith angle of $29^{\circ}$. The expected spectrum of Cas A from $0.3 \mathrm{TeV}$ to 1 $\mathrm{PeV}$ is shown in Fig. 3, and the parameters for the leptonic and hadronic model are listed in Tab. 2 and Tab. 3 respectively. According to the expectation results from Fig. 3, we can see that from $300 \mathrm{GeV}$ to $500 \mathrm{TeV}$, the statistic error of data obtained by LHAASO will be less than $10 \%$. Due to the Klein-Nishina effect, the spectral dominated by electrons is much softer than the hadronic acceleration above $10 \mathrm{TeV}$, and the expected result of LHAASO with a low statistic error can give an reasonable explanation on the high energy range. This estimation would be just sufficient to confirm whether the historical SNRs are PeVatron or not, and give the final judgement for the acceleration models.

\subsubsection{SNRs interacting with molecular clouds}

The massive molecular clouds located in the vicinity of SNRs, provide dense targets for hadronic interactions, and thus dramatically increase the chances of tracing the runaway protons via the secondary $\gamma$-rays. The location of molecular clouds close to SNRs could be accidental, but in general there is a deep link between SNRs and molecular clouds (Jiang et al. 2010), especially in the star-forming regions (Aharonian et al. 1996). SNRs interacting with molecular clouds are expected to strengthen $\pi^{0}$-decay emission and then could provide direct evidence of cosmic nuclei being accelerated at supernova shocks (Aharonian et al. 1994).

SNR IC 443 possesses strong molecular line emission regions that makes IC443 a case for an SNR interacting with molecular clouds. The X-ray emission of IC 443 is primarily thermal and peaked towards the interior of the northeast shell, indicating that IC 443 is a SNR with mix-morphology. Fermi (Abdo et al. 2010a) in the GeV region and VERITAS (Acciari et al.2009a), and MAGIC (Albert et al.2007a) in the $\mathrm{TeV}$ region up to $1 \mathrm{TeV}$ detected the $\gamma$-ray spectrum of IC 443, but at higher energy range, there is not yet observation, which is very important for determination on $\gamma$-ray emission mechanism.

W51C (G49.2-0.7) also interacts with the molecular clouds.
The W51 region was strong studied as it is known to host several objects. It contains three main components: two starforming regions W51A and W51B surrounded by very giant molecular cloud, and SNR W51C. W51C is a radio-bright SNR at a distance of $6 \mathrm{kpc}$ from Earth with an estimated age of $\sim 3 \times 10^{4} \mathrm{yr}$ (Koo et al. 1995). W51C is visible in X-rays showing both a shell type and center filled morphology. Shocked atomic and molecular gases have been observed, providing direct evidence on the interaction of W51C shock with a large molecular cloud (Reichardt et al. 2011; Aleksic et al. 2012). The GeV spectral result provided by Fermi indicates that leptonic model is difficult to explain $\gamma$-rays production and the most reasonable explanation is that hadronic interaction took place at the shocked shell of W51C emits GeV $\gamma$-rays (Abdo et al. 2009b). Moreover, MAGIC and H.E.S.S. also indicates the $\gamma$-ray emission from W51C tends to be dominated by $\pi^{0}$-decay up to several TeV (Fiasson et al. 2009; Reichardt et al. 2011; Aleksic et al. 2012). But this still has uncertainties for the acceleration mechanism above $10 \mathrm{TeV}$.

Besides of the leptonic model we described above, the accumulative diffusion model is also performed (in this paper, we call it AD model for short) (Li \& Chen 2010, 2012; Zhang \& Chen 2016). The AD model considers that the energetic protons colliding with the given molecular clouds are a collection of the diffusive protons escaping from the shock front as the SNR expands. A small distance between the SNR and molecular clouds is allowed ( $\mathrm{Li} \& \mathrm{Chen} 2010$ ). The distribution of escaping protons is assumed to be a power-law function, and the spectrum of the diffusive protons for any point near the SNR at any time is obtained. In the treatment of the dynamical evolution of a SNR, the Sedov-Taylor law is used for the adiabatic phase and the radiative phase. Besides, the finite-volume of the cloud in the vicinity of SNRs is considered. The $\gamma$-rays emitted from the secondary leptons are negligible compared with the dominant contribution of the protons themselves (Gabici et al. 2007, 2009).

The parameters for leptonic model are listed in Tab. 2, and the parameters for AD model are given in the published paper (Li \& Chen 2012). At the LHAASO site, the effective observation time is 6.53 hours per day for IC 443 and 6.0 hours per day for W51C with zenith angle less than $45^{\circ}$. IC 443 culminates with a zenith angle of $8^{\circ}$ and W51C culminates with a zenith angle of $16^{\circ}$. The expectation of LHAASO is given in Fig. 4, compared with the measurement of Fermi, MAGIC and VERITAS. From $300 \mathrm{GeV}$ to $500 \mathrm{TeV}$, the statistic error of data obtained by LHAASO will be less than $10 \%$. The discrepancy between the expectations from the two models will reach more than 5 sigma above $20 \mathrm{TeV}$. It indicates that LHAASO will make a great contribution on the accelerate measurement in the $\mathrm{TeV}$ range, providing the final judgement on leptonic or hadronic acceleration.

\subsection{Special case studies}

Most of unidentified $\mathrm{TeV}$ sources are extended $\gamma$-ray sources with SNRs, PWNs or PSRs in their region. The estimation of their significant should include the source extension during the calculation of background events. To model the source extension, a symmetric two-dimensional Gaussian shape is used for all extended sources in this work. The source extensions $\sigma_{\text {ext }}$ for each source is shown in Tab. 4.

We choose ARGO J2031+4157/Cygnus Cocoon as example to explore in more detail the spectral measurements at 
Tycho

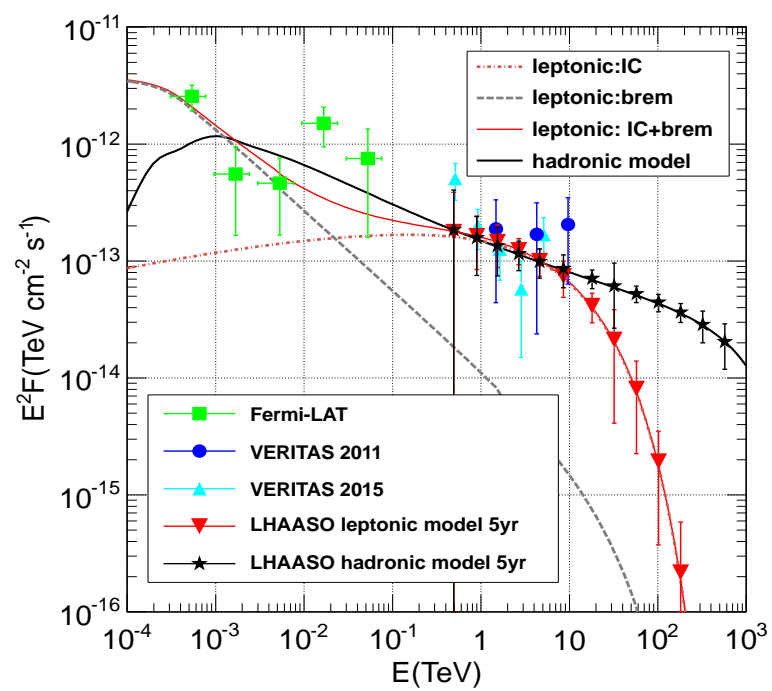

\section{Cassionpeia A}

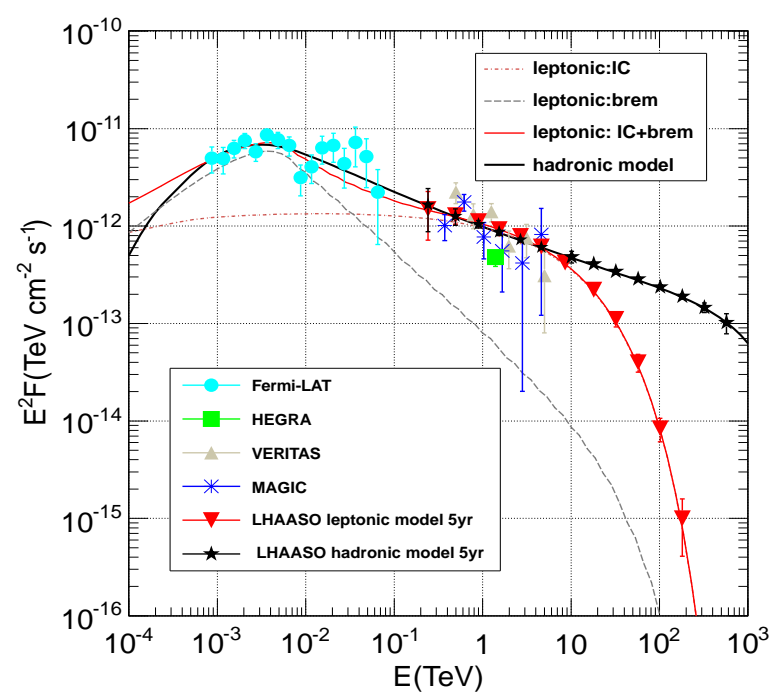

Figure 3. Expectation of the LHAASO project on the historical SNR spectrum. (A color version of this figure is available in the online journal.

\section{IC 443}

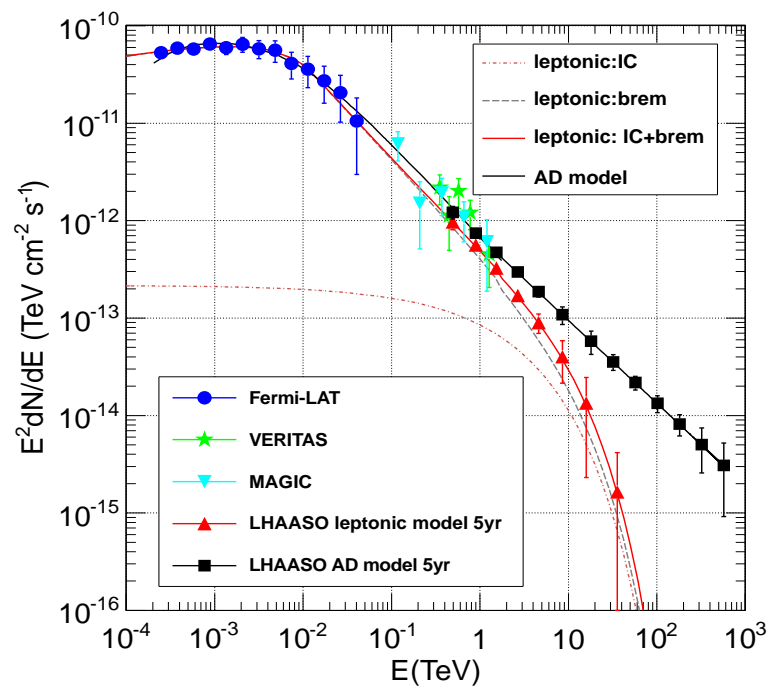

W51C

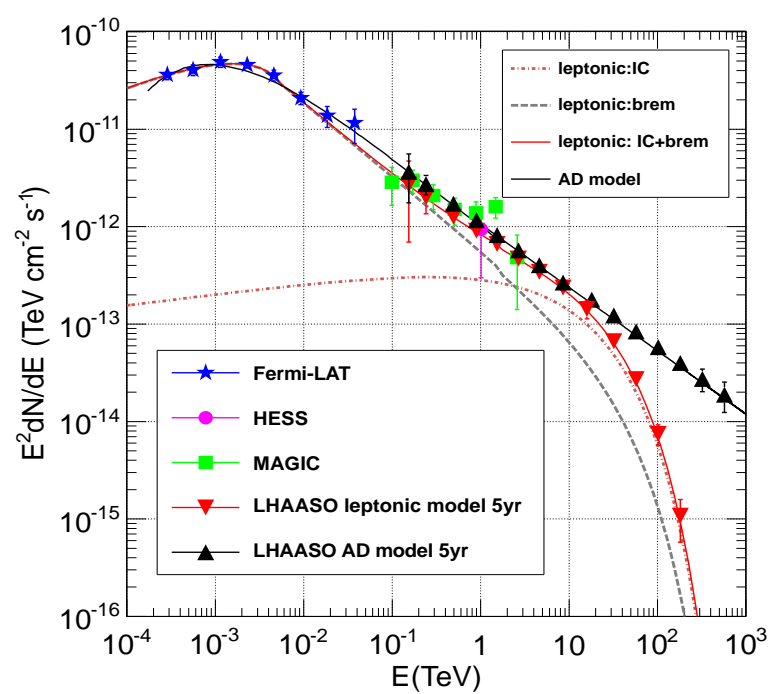

Figure 4. Expectation of the LHAASO project on SNRs interaction with molecular clouds spectrum. (A color version of this figure is available in the online journal.

high energy range. The Cygnus region of the Galactic plane, discovered by Fermi-LAT at GeV energies in the Cygnus superbubble, is the famous region in the northern sky for the complex features observed in radio, infrared, X-rays, and $\gamma$ rays. It contains a high density interstellar medium, rich in potential cosmic ray acceleration sites such as Wolf-Rayet stars, OB associations, and SNRs. This region is home of a number of $\mathrm{GeV} \gamma$-ray sources detected by Fermi-LAT (Nolan et al. 2012) and several noteworthy $\mathrm{TeV} \gamma$-ray sources detected by Milagro and ARGO-YBJ in the past decade. The Cygnus Cocoon, located in the star-forming region of Cygnus $X$, is interpreted as a cocoon of freshly accelerated cosmic rays related to the Cygnus super-bubble. The extended $\mathrm{TeV} \gamma$-ray source ARGO J2031+4157 (or MGRO J2031+41) is positionally consistent with the Cygnus Cocoon and another TeV source MGRO J2019+37 is a mysterious source only being detected by Milagro (Abdo et al. 2007a) above $20 \mathrm{TeV}$ and VERITAS (Aliu et al. 2014) above $1 \mathrm{TeV}$. The reason for the hard SED from such a spatially extended region is totally unknown. The discovery of this kind of sources and the more detailed multi-wavelength spectroscopic investigations can be an efficient way to explain the radiation mechanism of them.

At the LHAASO site, Cygnus Cocoon culminates with a zenith angle of $12^{\circ}$ and is observable for 7.2 hours per day. Fig. 5 shows all the spectral measurements by Fermi-LAT (Ackermann et al. 2011), ARGO-YBJ (Bartoli et al. 2014), Milagro (Abdo et al. 2007a b, 2009a), and the expectation results with LHAASO. The hadronic emission model with energy cuts off $150 \mathrm{TeV}$ is the maximum allowed by the ARGOYBJ upper limit. Taking Milagro data into account, the cut- 
Table 4

Table of a selection of UNID TeV $\gamma$-ray sourcs and a supperbubble (Cygnus Cocoon) shown with the expected significance above $10 \mathrm{TeV}$ using five years Monte Carlo simulation datas of LHAASO, assuming the sources are under the specific hypotheses for the energy spectrum. ("-" means the significance is less than $5 \sigma$.

\begin{tabular}{|c|c|c|c|c|c|c|c|c|}
\hline Name & R.A. & Dec. & $\overline{\alpha \alpha}$ & $\begin{array}{c}J_{0} \\
\left(\mathrm{TeV}^{-1} \mathrm{~cm}^{-2} \mathrm{~s}^{-1}\right) \\
\end{array}$ & $\begin{array}{l}\sigma_{e x t} \\
\left({ }^{\circ}\right) \\
\end{array}$ & $\begin{array}{c}\sigma \\
\left(E_{\mathrm{cut}}=100 \mathrm{TeV}\right) \\
\end{array}$ & $\begin{array}{c}\sigma^{\prime} \\
\left(E_{\mathrm{cut}}=30 \mathrm{TeV}\right)\end{array}$ & Ref. \\
\hline MAGIC J0223+403 & $02^{h} 23^{m} 12^{s}$ & $+43^{\circ} 00^{\prime}$ & $3.1 \pm 0.31$ & $4.07 \times 10^{-13}$ & 0 & - & - & 14 \\
\hline HESS J1832-093 & $18^{h} 32^{m} 50^{s}$ & $-09^{\circ} 22^{\prime}$ & $2.6 \pm 0.3$ & $4.8 \times 10^{-13}$ & 0 & 15.27 & 5.15 & 15 \\
\hline HESS J1834-087 & $18^{h} 34^{m} 45.6^{s}$ & $-08^{\circ} 45^{\prime}$ & $2.5 \pm 0.2$ & $3.7 \times 10^{-12}$ & 0.1 & 21.73 & 7.15 & 16 \\
\hline HESS J1841-055 & $18^{h} 40^{m} 55^{s}$ & $-05^{\circ} 33^{\prime}$ & $2.32 \pm 0.23$ & $3.76 \times 10^{-11}$ & 0.4 & 699.27 & 234.89 & 17 \\
\hline HESS J1857+026 & $18^{h} 57^{m} 11^{s}$ & $+02^{\circ} 40^{\prime}$ & $2.16 \pm 0.07$ & $5.37 \times 10^{-12}$ & 0.17 & 205.63 & 76.95 & 18 \\
\hline HESS J1858+020 & $18^{h} 58^{m} 20^{s}$ & $+02^{\circ} 05^{\prime}$ & $2.17 \pm 0.12$ & $6.0 \times 10^{-13}$ & 0.08 & 20.10 & 7.45 & 19 \\
\hline MGRO J1908+06 & $19^{h} 07^{m} 54^{s}$ & $+06^{\circ} 16^{\prime}$ & $2.54 \pm 0.36$ & $2.06 \times 10^{-11}$ & 0.49 & 220.80 & 97.13 & 20 \\
\hline VER J2016+371 & $20^{h} 16^{m} 02^{s}$ & $+37^{\circ} 11^{\prime}$ & $2.3 \pm 0.3$ & $3.1 \times 10^{-13}$ & 0 & 9.76 & 5.00 & 21 \\
\hline VER J2019+368 & $20^{h} 19^{m} 25^{s}$ & $+36^{\circ} 48^{\prime}$ & $1.75 \pm 0.08$ & $1.35 \times 10^{-12}$ & 2.0 & 58.36 & 22.40 & 22 \\
\hline VER J2019+407 & $20^{h} 20^{m} 04.8^{s}$ & $+40^{\circ} 45^{\prime}$ & $2.37 \pm 0.14$ & $1.5 \times 10^{-12}$ & 0.23 & 38.74 & 17.89 & 23 \\
\hline ARGO J2031+4157 & $20^{h} 31^{m} 12^{s}$ & $+42^{\circ} 30^{\prime}$ & $2.6 \pm 0.3$ & $3.05 \times 10^{-12}$ & 2.0 & 10.86 & 5.3 & 24 \\
\hline
\end{tabular}

Notes: Columns from left to right are: source name, R.A., Declination, spectral index, flux normalization, cutoff energy, expected significance by LHAASO (for $E_{\text {cut }}=100 \mathrm{TeV}$ if the cutoff energy has not been measured), expected significance by LHAASO for $E_{\text {cut }}=10 \mathrm{TeV}$, and the references of the measurements. References: (14) Aliu et al. (2009); (15) Abramowski et al. (2015); (16) Albert et al. (2006); (17) Bartoli et al. (2013); (18) Aleksic et al. (2014); (19) Aharonian et al. (2008a); (20) Bartoli et al. (2012); (21) Aliu et al. (2014); (22) Aliu et al. (2014); (23) Aliu et al. (2013); (24) Bartoli et al. (2014);

\section{Cygnus Cocoon}

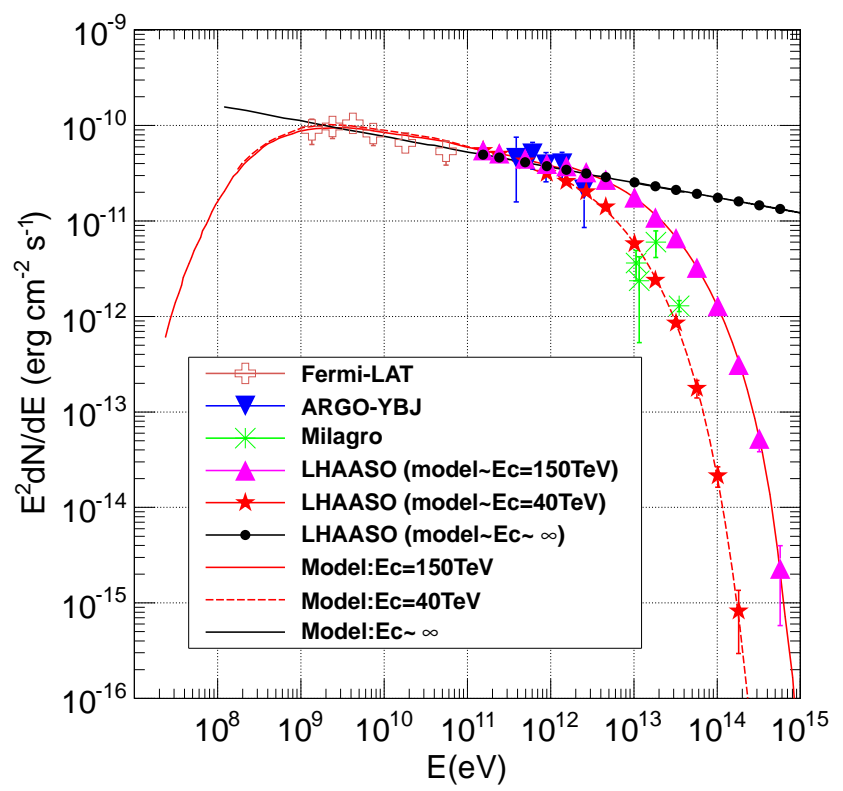

Figure 5. Expectation of the LHAASO project on Cygnus Cocoon by using one year MC data, compared with the measurement of FermiLAT(Ackermann et al. 2011), ARGO-YBJ(Bartoli et al. 2014), Milagro(Abdo et al. 2007a; 2007b; 2009a). (A color version of this figure is available in the online journal.)

off energy would be around $40 \mathrm{TeV}$. One year observation of LHAASO will be sufficient to give a judgement on the different cutoff energy models from $300 \mathrm{GeV}$ to several hundreds $\mathrm{TeV}$. Besides, if the spectrum has no cutoff energy shown in Fig. 5 (black dots), LHAASO will give an accurate measurement up to PeV. It will provide important information for investigating the particle acceleration within the super-bubble.

\section{CONCLUSIONS}

With a sensitivity of 10 mili-Crab at $50 \mathrm{TeV}$, LHAASO will launch the most accurate detection of $\gamma$-ray spectrum of the
SNRs in the energy region above $30 \mathrm{TeV}$. In the field of view of LHAASO, 8 identified GeV-TeV or TeV SNRs observed by previous experiments will be detected by LHAASO with precise spectrum unprecedented extended up to few hundred $\mathrm{TeV}$. Besides, LHAASO has potential to discover more $\mathrm{TeV}$ SNRs which has not yet observation at $\mathrm{GeV}$ region and can also provide important information on unidentified $\mathrm{TeV} \gamma$ ray sources with a high significance. According to the detailed research on the spectrum, different theoretical models can obtain the effective tests by observation of LHAASO. Especially, with the high sensitivity of LHAASO above $30 \mathrm{TeV}$, it is expected that LHAASO is more sensitive to different leptonic or hadronic models of SNR $\gamma$-ray emission. High statistics and accurate measurement provided by LHAASO will sufficiently offer a final judgement between different SNR theoretical models, to reveal cosmic ray acceleration mechanism in Galaxy.

\section{ACKNOWLEDGMENTS}

We acknowledge the supports of the NSFC grants (No.11205165, No.11575203, No.11375052, No.11375210, No.11233001) and CAS (U1532258), the Program for New Century Excellent Talents in University (NCET-13-0342), the Shandong Natural Science Funds for Distinguished Young Scholar (JQ201402) and 973 Program grant 2015CB857100.

\section{REFERENCES}

Abdo, A. A., et al., 2007a, ApJ, 658, L33 Abdo, A. A., et al., 2007b, ApJ, 664, L91 Abdo, A. A., et al., 2009a, ApJ, 700, L127 Abdo, A. A., et al. 2009b, ApJ, 706, L1 Abdo, A. A., et al. 2010a, ApJ, 712, 459 Abdo, A. A., et al. 2010b, ApJL, 710, L92 Abdo, A. A., et al. 2011, ApJ, 734, 28

Abramowski, A., et al. 2015, MNRAS, 446, 1163

Acciari, V. A., et al. 2009a, ApJ, 698, L133

Acciari, V. A., et al. 2009b, ApJ, 703, L6

Acciari, V. A., et al. 2010, ApJ, 714, 163

Acciari, V. A., et al., 2011, ApJL, 730, L20

Acero, F., et al. 2010, A\&A, 516, A62

Acero, F., et al. 2015a, ApJ, 218, 23

Acero, F., et al. 2015b, A\&A, 580, A74 
Ackermann, M., Ajello, M., Allafort, A., et al., 2011, Sci., 334, 1103 Ackermann, M., Ajello, M., Allafort, A., et al., 2013, Sci, 339, 807

Aharonian, F., Drury, L. O. C., \& Volk, H. J., 1994, A\&A, 285, 645

Aharonian, F., \& Atoyan, A. M. , 1996, A\&A, 309, 917

Aharonian, F., et al. 2001, A\&A, 370, 112

Aharonian, F., et al. 2006, A\&A, 460, 365

Aharonian, F., Akhperjanian, A. G. , et al. 2008a, A\&A, 477, 353

Aharonian, F., Akhperjanian, A. G. , et al. 2008b, A\&A, 484, 435

Aharonian, F., 2013, Astropar.Phys., 43, 71

Albert, J., et al., 2006, ApJ, 643, L53

Albert, J., et al. 2007a, ApJ, 664, L87

Albert, J., et al. 2007b, A\&A, 474, 937

Aleksic, J., Alvarez, E. A., et al. 2012, A\&A, 541, A13

Aleksic, J., et al. 2014, A\&A, 571, A96

Aliu, E., et al., 2009, ApJ, 692, L29

Aliu, E., et al., 2013, ApJ, 770, 93

Aliu, E., et al., 2014, ApJ, 788, 78

Araya, M., \& Frutos, F., 2012, MNRAS, 425, 2810

Bamba, A., Yamazaki, R., Yoshida, T., Terasawa, T., \& Koyama, K., 2005, ApJ, 621, 793

Bartoli, B., et al., 2012, ApJ, 760, 110

Bartoli, B., et al., 2013, ApJ, 767, 99

Bartoli, B., et al., 2014, ApJ, 790, 152.

Bartoli, B., et al., 2015, arXiv:1511.06851

Bell, A. R.,1978a, MNRAS, 182, 147

Bell, A. R.,1978b, MNRAS, 182, 443

Bell, A. R., 2004, MNRAS, 353, 550

Blandford, R., \& Cowie, L. L., 1982, ApJ, 260, 625

Blandford, R., \& Eichler, D., 1987, Physics Reports, 1154, 1

Blumer, J., Engel, R., \& Horandel, Jorg R., 2009, Progress in Particle and Nuclear Physics, 63, 293

Cao, Z., 2010, CPC, 34, 249

Chen, S. Z., 2013, Sci China-Phys Mech Astron, 56, 1454

Cui, S. W., Liu, Y., Liu, Y. J., \& Ma, X.H., 2014, Astropar.Phys., 54, 86

Dickel, J. R., van Breugel, W. J. M., \& Strom, R. G., 1991, AJ, 101, 2151

Drury, L. O. C., 1983, Rep.Prog.Phys., 46, 973

Drury, L. O. C., Aharonian, F., \& Volk, H. J., 1994, A\&A, 287, 959

Ellison, D. C., Patnaude, D. J., Slane, P., \& Raymond, J., 2010, ApJ, 712, 287

Fiasson, A., Marandon, V., Chaves, R., \& Tibolla, O. 2009, in Proc. 31st International Cosmic Ray Conference, in press

Francois Brun, Mathieu de Naurois et al. 2011, arXiv:1104.5003

Gabici, S., Aharonia, F., \& Blasi, P., 2007, Ap\&SS, 309, 365

Gabici, S., Aharonia, F., \& Casanova, S., 2009, MNRAS, 396, 1629

Giordano, F., et al. 2012, ApJL, 744, L2

Giuliani, A., et al. 2011, ApJL, 742, L30
Hanbury Brown, R. \& Hazard, C., MNRAS, 1953, 113, 123

Hartman, R. C., et al. 1999, ApJS, 123, 79

He, H. H., 2015, in Proc. 34th International Cosmic Ray Conference

Holder, J., 2012, Astropar.Phys., 39, 61

Horandel, Jorg R., 2003, Astropar.Phys., 19, 193

Hwang, U., Petre, R., Szymkowiak, A. E., \& Holt, S. S., 2002, Journal of

Astrophysics and Astronomy, 23, 81

Ivanenko, L. P., 1988, JETP Lett., 48, 9

Jiang, B., Chen, Y., Wang, J.Z., Su, Y., Zhou, X., Safi-Harb, s., \& Delaney, T., 2010, ApJ, 712, 1147

Joncas, G., Higgs, L. A., 1990, A\&AS, 82, 113

Katsuda, S., Petre, R., Hughes, J. P., Hwang, U., et al. 2010, ApJ, 709, 1387

Kelner, S. R., Aharonian, F., \& Bugayov, V. V., 2006, Phys.Rev.D, 74, 034018

Koo, B. C., Kim, K. T., \& Seward, F. D., 1995, ApJ, 447, 211

Li, H., \& Chen, Y. 2010, MNRAS, 409, 35

Li, H., \& Chen, Y. 2012, MNRAS, 421, 935

Li, T. P., \& Ma, Y. Q. 1983, ApJ, 272, 317

Li, H., Liu, S. M., \& Chen, Y. 2011, ApJL, 742, L10

Mao, Z., \& Yu, Y., W., 2013, Res.Astron.Astrophys., 13, 8, 952

Nolan, P. L., Abdo, A. A., Ackermann, M., et al. 2012, ApJS, 199, 31

Park, N., et al. 2015, in Proc. 34th International Cosmic Ray Conference

Pineault, S., \& Joncas, G. 2000, AJ, 120, 3218

I. Reichardt et al., 2011, Mem.S.A.It., 82, 735

Schure, K. M., Bell, A. R., Drury, L. O. C., \& Bykov, 2012, Space Sci Rev, 173,491

Slane, P. et al. 2014, ApJ, 783, 33

Wendy Stroman, \& Martin Pohl, 2009, ApJ, 696, 1864

Tanaka, T., Allafort, A.,Ballet, J. et al. 2011, ApJL, 740, L51

Uchiyama, Y., Blandford, R., Funk, S., Tajima, H. \& Tanaka, T., 2010, ApJL, 723, L122

Yang, R., Z., Zhang, X., Yuan, Q., \& Liu, S., M., 2014, A\&A, 567, A23

Yao, Z., G., Zha, M., Cao, Z., 2009, in Proc. 31st International Cosmic Ray Conference, in press

Yao, Z., G., Wu, H., R., Chen, M., J., et al., 2011, in Proc. 32nd International Cosmic Ray Conference, in press

Yuan, Q., Liu, S. M., Bi, X. J. et al. 2011, ApJ, 735, 120

Yuan, Q., Liu, S. M., \& Bi, X. J. 2012, ApJ, 761, 133

Yuan, Q., Huang, X. Y., Liu, S., M., \& Zhang, B., 2014, ApJL, 785, L22

Yuan, Y. J., Funk, S., Johannesson, G., Landae, J., Tibaldo, L., \&

Uchiyamaet, Y., 2013, ApJ, 779, 117

Zhang, X., Chen, Y., Li, H., \& Zhou, X., 2013, MNRAS, 429, L25

Zhang, X., \& Chen, Y.,2016, ApJ, 821, 43

Zhao, Y., Yuan, Q., Bi, X., J., Zhu, F., R., \& Jia, H., Y., 2016,

Int.J.Mod.Phys.D, 25, 1, 1650006

Zirakashvili, V., N. et al, 2014, ApJ, 785, 130 